\title{
Comparison Between Analgesic Effect of Dexamethasone and Dexmedetomidine as an Adjuvant to Bupivacaine for Spinal Anaesthesia for Elective Caesarean Sections
}

\author{
Baher Said Abdelhady*, Tamer M. Allam and Yehya. Sh. Dabour \\ Lecturer of Anaesthesiology and intensive Care, Faculty of medicine, Benha University, Benha, Egypt \\ *Corresponding author: Baher Said Abdelhady, Lecturer of Anaesthesiology and intensive Care, Faculty of medicine, Benha University, \\ Benha, Egypt
}

Received date: 01 February, 2022 |

Accepted date: 14 February, 2022 |

Published date: 17 February, 2022

Citation: Abdelhady BS, Allam TM and Sh. Dabour Y. (2022) Comparison between analgesic effect of dexamethasone and dexmedetomidine as an adjuvant to bupivacaine for spinal anaesthesia for elective caesarean sections. J Anaesth Anesth Drug 2(1): doi https://doi.org/10.54289/JAAD2200102

Copyright: () 2022 Abdelhady BS, et al. This is an open-access article distributed under the terms of the Creative Commons Attribution License, which permits unrestricted use, distribution, and reproduction in any medium, provided the original author and source are credited.

\begin{abstract}
Background

Caesarean sections are usually performed under spinal anaesthesia. It provides effective postoperative pain control over a limited period of time. As a result, various intrathecal additives have been used. Recently, dexamethasone and dexmedetomidine have been used as adjuncts for different types of regional anaesthesia without increasing the risk of neurological complications.
\end{abstract}

Objectives: We compared the analgesic effects of intrathecal dexamethasone and dexmedetomidine in elective caesarean sections.

Design: A single-blind randomized trial.

Setting: Benha University Hospital, between February 2021 and July 2021.

Patients: We randomly enrolled 633 pregnant women in this study. Only 580 patients met all the inclusion and exclusion criteria. The inclusion criteria were age of 18 to 40 years old, an ASA class II - III, gestational age > 37 weeks, BMI less than $30 \mathrm{~kg} / \mathrm{m} 2$ who were electively scheduled for. The exclusion criteria included patient refusal, age $<18$ or $>40$ years, known allergy to medications used in the study, BMI more than $30 \mathrm{~kg} / \mathrm{m} 2$, coagulopathies or anticoagulant use, cerebrovascular insufficiency, neuromuscular diseases and diabetic neuropathy.

Intervention: The dexmedetomidine group (A) received $10 \mu \mathrm{g}$ of dexmedetomidine with $12.5 \mathrm{mg}$ hyperbaric bupivacaine $0.5 \%$ intrathecally $(\mathrm{n}=290)$. The dexamethasone group $(\mathrm{B})$ received $4 \mathrm{mg}$ of dexamethasone with $12.5 \mathrm{mg}$ hyperbaric bupivacaine $0.5 \%$ intrathecally $(\mathrm{n}=290)$.

Main outcome: The time to initial analgesic rescue (pethidine $0.5 \mathrm{mg} / \mathrm{kg}$ ) and the duration of motor blockage were assessed. Results: The time to the first rescue analgesia and motor blockade were significantly higher in the dexmedetomidine group. (418 min vs $190 \mathrm{~min}$ ), $\mathrm{P}<0.001$, (324 $\mathrm{min}$ vs $144 \mathrm{~min}$ ), $\mathrm{P}<0.001$ respectively. 
Conclusion: According to this study, both drugs could be safely added to bupivacaine intrathecally. Dexmedetomidine provides significantly longer postoperative analgesia and motor blockade times than dexamethasone. Further dose-response studies are needed to determine the optimum dosage of dexamethasone and dexmedetomidine and the safety of the intrathecal route.

\section{Key Points:}

- Spinal anaesthesia provides effective post operative analgesia; however, it is for a limited period of time.

- To provide appropriate postoperative pain management in addition to Cope with the issue of short duration of action of local anaesthetics, several adjuvants have been used.

- Recently, dexamethasone and dexmedetomidine have been used as adjuncts for different types of regional anaesthesia without increasing the risk of neurological complications.

- We designed a single-blind randomized study to compare the analgesic effect of intrathecal dexamethasone and dexmedetomidine in elective caesarean sections.

- Intrathecal administration of dexamethasone and dexmedetomidine appears to be safe and provides prolonged duration of analgesia with the superiority of dexmedetomidine; however, further studies are required to approve intrathecal route of These drugs or to refuse it.

\section{Introduction}

Spinal anaesthesia is the most commonly used anaesthesia for caesarean sections. When no contraindications to this technique exist, it is an ideal option [1]. It avoids general anaesthesia risks such as maternal awareness, aspiration of gastric contents, infant respiratory and difficulty with airway management $[2,3]$. The most prevalent side effect of spinal anaesthesia is hypotension, which is directly associated with maternal and foetal morbidity and mortality. Several studies have shown that decreasing the intrathecal local anesthetic dose can reduce the frequency of spinal-induced hypotension while maintaining a relatively short period of anaesthesia and analgesia [3].

Appropriate postoperative pain control has many advantages including patient comfort, earlier mobilization, fewer pulmonary and cardiac problems, a lower risk of deep vein thrombosis, a lower likelihood of developing of neuropathic pain, and a lower cost of health care. As a result, expanding the duration of local anaesthesia is usually a focus of interest for anesthesiologists to produce extended and effective postoperative analgesia [4].

To address the issue of limited duration of action of local anaesthetics, a variety of additives are used to extend the duration of spinal anaesthesia, such as epinephrine, phenylephrine, clonidine, opioids; however, these adjuvants are associated with undesired side effects [3].

Recently, dexamethasone $[\mathbf{1 , 5 , 6 ]}$ and dexmedetomidine [1014] have been used as adjuncts for different types of regional anaesthesia without increasing the risk of neurological complications.

A previous study compared intrathecal dexamethasone and dexmedetomidine in lower abdominal surgeries and showed that dexmedetomidine resulted in longer duration of sensory block, motor block, and postoperative analgesia [15].

Considering a number of studies comparing dexmedetomidine and dexamethasone as adjuvants for bupivacaine in spinal anaesthesia, we designed a single-blind randomised study to compare the analgesic effect of intrathecal dexamethasone and dexmedetomidine in elective caesarean sections.

\section{Material and Methods}

Ethics

Ethical approval for this study (Rc 5-/2-2020) was provided by the Institutional Research Ethics Committee of the Faculty of Medicine, Benha University, Benha, Egypt (chairperson Prof. Ibrahim S. Elgendy) on 18/06/2020 and was performed 
at Benha University Surgical Hospital, Egypt.

\section{Patients}

Patient enrolment was conducted from Feb2021 to July 2021. Written informed consent was obtained from each patient who participated in the trial during the preoperative visit. This manuscript adheres to the CONSORT guidelines. We enrolled female patients (18 to 40 years old) with an ASA class II - III, gestational age > 37 weeks, BMI less than 30 $\mathrm{kg} / \mathrm{m}^{2}$ who were electively scheduled for caesarean section using spinal anaesthesia. The exclusion criteria included patient refusal, age $<18$ or $>40$ years, known allergy to medications used in the study, BMI more than $30 \mathrm{~kg} / \mathrm{m}^{2}$, coagulopathies or anticoagulant use, cerebrovascular insufficiency, neuromuscular diseases, diabetic neuropathy, and psychiatric disorders.

This trial comprised 580 pregnant patients who met all the inclusion and exclusion criteria and were randomly assigned to 2 equal groups, each consisting of 290 patients, namely group A (dexmedetomidine group) and group B (dexamethasone group). Patients in group A received $10 \mu \mathrm{g}$ dexmedetomidine with $12.5 \mathrm{mg}$ hyperbaric bupivacaine $0.5 \%$ intrathecally. Patients in group B received $4 \mathrm{mg}$ dexamethasone with $12.5 \mathrm{mg}$ hyperbaric bupivacaine $0.5 \%$ intrathecally.

The time to initial analgesic rescue (pethidine $0.5 \mathrm{mg} / \mathrm{kg}$ ) and duration of motor blockage were assessed. The incidence of shivering was recorded every $15 \mathrm{~min}$ intraoperatively.

\section{Anaesthesia}

Each patient underwent a- regular preoperative assessment, which included a- routine medical history, physical examination, and laboratory testing (complete blood picture, kidney function tests, liver function tests and prothrombin time). Premedications were not administered to the patients. All patients were received $10 \mathrm{ml} / \mathrm{kg}$ of lactated Ringer's solution via an intravenous cannula of 18 gauge and were monitored with five lead ECG, pulse oximetry, and noninvasive blood pressure (NIBP) every 5 min intraoperatively. A local anaesthetic (3 $\mathrm{ml}$ of lidocaine 2 percent) was administered under a full aseptic procedure at the site of spinal injection. In the sitting position, a 27 gauge (Quincke needle) was used for the subarachnoid block, which was applied at the L3-L4/L4-L5 space. Following completion of the spinal block, patients were immediately placed in the supine position. The sensory blockade was assessed using a pin prick method with a 25 gauge needle every 2 min until the greatest level was reached, and then every 15 min until a- two segment regression of the block occurred. Motor block was measured at the same time intervals using a modified Bromage scale $(0=$ no paralysis, $1=$ unable to elevate extended legs, 2 = unable to flex the knee, 3 = unable to flex the ankle); (Moeen \& Moeen, 2017). The time to the first analgesic rescue (pethidine $0.5 \mathrm{mg} / \mathrm{kg}$ ) and the duration of motor blockage were also recorded. The incidence of shivering was registered every 15 min during surgery.

\section{Statistical Analysis}

Data management and statistical analyses were performed using SPSS version 25 software. (IBM, Armonk, New York, USA). The normality of the numerical data was assessed using the Shapiro-Wilk test. Numerical data were summarised as means and standard deviations, and categorical data were summarised as numbers and percentages. Comparisons between both groups were performed using independent t-test for numerical data. All $\mathrm{P}$ values were two-sided. $\mathrm{P}$ values less than 0.05 were considered significant. The sample size was calculated using $G^{*}$ Power software version 3.1.9.2 based on an expected small to medium effect size between both groups regarding time to $1^{\text {st }}$ analgesia. The total sample size was 580 patients (290 patient in each group). Alpha and power were adjusted to 0.05 and 0.95 , respectively.

\section{Results}

\section{Demographic data:}

no significant differences were noted between the groups regarding age $(P=0.187)$ and $\mathrm{BMI}(\mathrm{P}=0.120)$. All participants in our study were ASA class II (Table 1)

\section{Assessment of analgesia and motor block:}

The time to the first rescue analgesia was significantly higher in the dexmedetomidine group (418 $\mathrm{min}$ ) than in the dexamethasone group (190 min), P < 0.001). In addtion, motor block was significantly higher in the dexmedetomidine group (324 min) thanin the dexamethasone group (144 min), $\mathrm{P}<0.001$ ). (Table 2, Figure 1) 
Table1: Demographic variables

\begin{tabular}{|l|l|l|l|l|}
\hline & & Dexamethasone $(\mathbf{n}=\mathbf{2 9 0})$ & Dexmedetomidine $(\mathbf{n}=\mathbf{2 9 0})$ & P value \\
\hline Age (years) & Mean \pm SD & $31 \pm 6$ & $31 \pm 6$ & 0.187 \\
\hline BMI (kg/m2) & Mean \pm SD & $28.4 \pm 1.7$ & $28.7 \pm 1.7$ & 0.120 \\
\hline
\end{tabular}

Tabel 2: Assessment of analgesia and motor block

\begin{tabular}{|l|l|l|l|l|}
\hline & & Dexamethasone $(\mathbf{n}=\mathbf{2 9 0})$ & Dexmedetomidine (n= 290) & P value \\
\hline Motor block (min) & Mean \pm SD & $144 \pm 90$ & $324 \pm 112$ & $<0.001$ \\
\hline Time to first rescue analgesia (min) & Mean \pm SD & $190 \pm 35$ & $418 \pm 133$ & $<0.001$ \\
\hline
\end{tabular}

Figure (1)

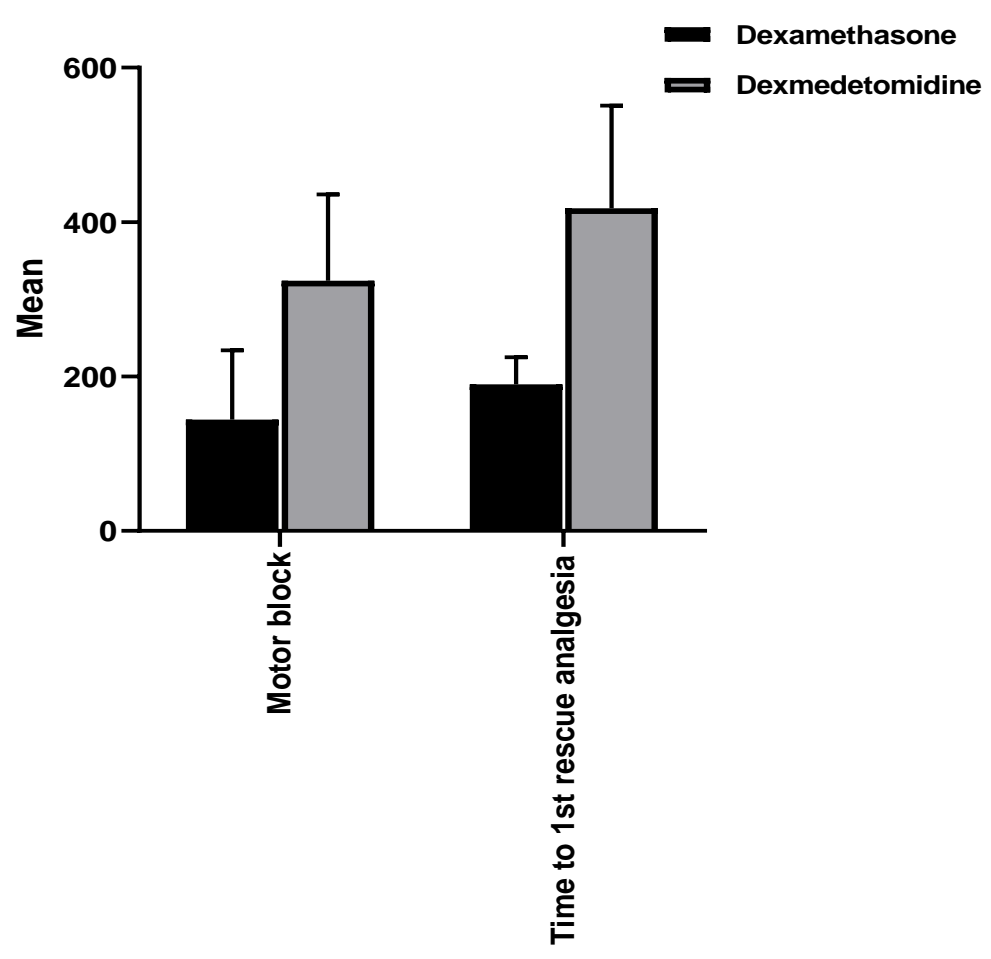

\section{Figure legend}

Figure (1) demonstrates comparison between dexamethasone and dexmedetomidine regarding duration of analgesia and motor blockade:

A) motor blockade B) duration of analgesia

\section{Assessment for intraoperative shivering}

None of the participants experienced shivering

\section{Discussion}

Anaesthesiologists are frequently concerned about increasing the duration of local anaesthetics in order to achieve extended and effective postoperative analgesia [4]. Many authors have used dexmedetomidine $[\mathbf{3 , 1 0 - 1 3 , 1 7 ]}$ and dexamethasone $[1,7,19,33]$ as adjuvants to local anaesthetics and have reported significant results; however, there has been little agreement and limited evidence about their safety and ideal doses.

Dexmedetomidine is a selective alpha-adrenoreceptor agonist. It has the capability to prolong the period of analgesia and anaesthesia in neuroaxial and peripheral anaesthesia. $[3,10-13,17]$. Moreover, it is not associated with respiratory depression [21]. There was no evidence of any neurological deficit associated with intrathecal dexmedetomidine in a preclinical trial or in clinical practice [19].

Dexamethasone possesses anti-inflammatory and analgesic 
properties through suppression of nociceptive C-fibre neural discharges. Dexamethasone may affect intraspinal prostaglandin synthesis when administered intrathecally. The precise mechanism of the analgesic action is still unknown [15]. Intrathecal dexamethasone has shown promising results. Few studies have found that intrathecal administration of dexamethasone extends the duration of sensory block and improves postoperative analgesia without causing any obvious complications. [1, 7, 19, 23-25, 33-35]. This outcome was consistent with preclinical findings [8] as well as a prior case series of 200 patients who underwent 2000 intrathecal injections of dexamethasone $8 \mathrm{mg}$ to treat posttraumatic visual impairment, which revealed no neurologic sequelae [9]. Additionally, several studies have reported analgesic effect of epidural dexamethasone [37-39].

In this trial, we observed that adding dexmedetomidine to bupivacaine for spinal anaesthesia resulted in a significantly longer duration of motor block and postoperative analgesia than the addition dexamethasone to intrathecal bupivacaine, with no evident adverse effects. The mean duration of postoperative analgesia for dexmedetomidine was $(418 \pm 133 \mathrm{~min})$ which is similar to the results of a meta-analysis of neuraxial dexmedetomidine, which revealed that the mean duration of postoperative analgesia was increased by approximate 420 minutes [11].

Furthermore, our findings are consistent with those of Elzayyat [15], Hassan [21] and Gonapa [27] who found the same significant differences between the two adjuvants. Elzayyat et al [15]. compared intrathecal dexmedetomidine and dexamethasone as additives to intrathecal bupivacaine in lower abdominal surgery in 60 adult patients and found that dexmedetomidine provided a longer duration of motor block, and longer postoperative analgesia [15]. Time to first analgesic in dexmedetomidine group was $(4.02 \pm 1.21)$ hours compared to dexamethasone group which was $(2.80 \pm 0.86)$ hours. Hassan et al [21] compared intrathecal dexmedetomidine and dexamethasone as adjuvants to intrathecal bupivacaine anaesthesia in lower limb orthopaedic surgery in 60 adult patients. They found that dexmedetomidine provided longer duration of spinal anaesthesia and longer postoperative analgesia [21]. Time to first analgesic requested in dexmedetomidine group was
(293.50 \pm 15.57$)$ minutes compared to dexamthasone group

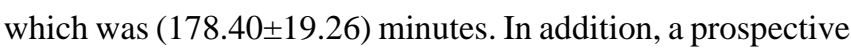
randomised study conducted by Gonapa [27] et al. compared dexmedetomidine and dexamethasone as adjuncts to bupivacaine for caudal analgesia during lower abdominal surgeries in paediatric patients and reported that dexmedetomidine was associated with longer postoperative analgesia [27]. In addition, dexmedetomidine offers longer postoperative analgesia than dexamethasone in various forms of regional anaesthesia, according to several trials published in the literature $[\mathbf{2 2 , 2 6 , 2 9 , 3 0 ]}$. Thakur et al. examined dexmedetomidine and dexamethasone as adjuncts to bupivacaine in transverses abdominis plane block for postoperative analgesia after caesarean delivery with the subarachnoid block. They found that adding dexmedetomidine and dexamethasone to bupivacaine lowered postoperative pain, lengthened analgesia, and minimised the need for further analgesics with an extended period of analgesia in the dexmedetomidine group [29]. Mazy et al. studied dexamethasone and dexmedetomidine as adjuncts to preperitoneal bupivacaine for postoperative analgesia in caesarean sections and dexmedetomidine was found to produce superior analgesia than dexamethasone [26]. Sana designed a randomised, prospective, doubleblinded and controlled study to examine the analgesic effect of the addition of dexmedetomidine and dexamethasone to bupivacaine during fascia iliaca block during surgeries of proximal femoral fracture. They demonstrated that the addition of dexmedetomidine provided better postoperative analgesia than dexamethasone [30]. Gao et al compared the effect of dexmedetomidine and dexamethasone as adjuvants for ropivacaine for erector spinae plane block in videoassisted thoracoscopic lobectomy and found that dexmedetomidine had a prolonged sensory block period with effective acute pain management, and fewer rescue analgesics than dexamethasone [22].

However, some studies appear contradictory. In a study by Ismaiel et al, dexmedetomidine and dexamethasone were compared as intrathecal adjuvants to prevent perioperative shivering in caesarean sections. They noted that dexamethasone resulted in longer postoperative analgesia, while dexmedetomidine was associated with prolonged 
motor block [20]. This contrary might be explained by the different doses of both adjuvants, as in Ismaiel's [20] study, dexmedetomidine was administered at a dose of $5 \mathrm{ug}$, and dexamethasone was administered at a dose $8 \mathrm{mg}$. Similarly, a recent meta-analysis reported that dexamethasone seems to be better than dexmedetomidine as a perineural adjuvant for supraclavicular brachial plexus block [14]. However, a recent meta-analysis [28] compared the analgesic effect of dexamethasone and dexmedetomidine in peripheral nerve blocks in patients who underwent surgery under regional or combined regional and general anaesthesia and found that both drugs had equivalent analgesic effects but with lowquality evidence; furthermore, because the included trials were highly heterogeneous, this meta-analysis showed a significant flaw. However, these meta-analyses were conducted on peripheral nerve blocks rather than on subarachnoid blocks.

Intrathecal dexmedetomidine significantly prolonged motor blockage, potentially delaying rehabilitation and extra attention to patients is required in the early postoperative stage to minimise accidental falls.

In our study, the dose of dexmedetomidine(10 ug) was in line with a recent prospective randomised double-blind doseresponse trial [31] that concluded that $10 \mu \mathrm{g}$ of intrathecal dexmedetomidine compared to lesser doses as an adjuvant to bupivacaine considerably increased the duration of sensory block, motor block, and analgesia without any noticeable increase in the incidence of adverse effects, while the dose of dexamethasone $(8 \mathrm{mg}$ ) was in agreement with a recent metaanalysis, which included 2138 patients, demonstrating a ceiling dose of $4 \mathrm{mg}$ for perineural dexamethasone with very low-quality evidence [5].

Interestingly, none of the participants had experienced shivering intraoperatively. This demonstrates the role of dexmedetomidine and dexamethasone in the prevention of shivering, as reported in a previous meta-analysis which showed the role of dexmedetomidine in the prevention of shivering in caesarean sections when added to spinal anaesthesia [36]. Furthermore, our findings match those of Ismaiel [20], who found dexmedetomidine to be as effective as dexamethasone in the prevention of shivering. Additionally, Moeen and Moeen [7] in their study demonstrated that intrathecal meperidine, effectively controlled post-spinal shivering with fewer side effects. Several studies have shown that dexmedetomidine alleviates shivering effects by mediating thermoregulatory inhibition through alpha 2 - adreno receptors, which are distributed hypothalamus [36]. Other studies have confirmed that dexmedetomidine directly increases the temperature range without affecting thermoregulatory defences, thereby reducing the incidence of shivering [36].

The lack of homogeneity in dosages, along with a shortage of publications, makes it impossible to make recommendations at the moment. More dose-response studies are needed to determine the appropriate doses of dexamethasone and dexmedetomidine, as well as to assess the safety of the intrathecal route. In other words, standardised studies are required to approve or refuse the intrathecal route of these drugs.

\section{Limitations}

A small sample size, little evidence regarding optimal doses and side effects such as hypotension and bradycardia were not evaluated. Furthermore, PONV was not investigated in addition to postoperative shivering assessment.

\section{Conclusion}

According to this study, both drugs could be safely added to bupivacaine intrathecally. Dexmedetomidine provides significantly longer postoperative analgesia and motor blockade time than dexamethasone. Additionally, both drugs can prevent intraoperative shivering. More dose-response studies are needed to determine the optimum dosage of dexamethasone and dexmedetomidine, as well as to assess the safety of the intrathecal route.

\section{Acknowledgements:}

1. Assistance with article: we thank Asmaa Elbab (M.Sc.), Benha faculty of Medicine, Benha, Ziad Mohsen (M.Sc.), Benha faculty of Medicine, Benha and Nermmen Nabil (M.Sc.), Benha faculty of Medicine ,Benha for their help in obtaining consents from patients.

2. Financial support and sponsorship: none declared

3. Conflict of interest: none declared

4. Presentation: none declared 


\section{References}

1. Tkachenko R and Pyasetska N. (2019) ESRA19-0295 The efficiency of intrathecal dexamethasone for spinal anesthesia in elective caesarean section. Regional Anesthesia \& Pain Medicine. 44: A192-A193.

2. Pyasetska N. (2020) The Efficacy of Intrathecal Dexamethasone to Prevent Early Complications of Spinal Anesthesia for Elective Caesarean Section". Technology Transfer: Innovative Solutions in Medicine. 10-13.

3. Xia F, Chang X, Zhang Y, Wang L \& Xiao F. (2018) The effect of intrathecal dexmedetomidine on the dose requirement of hyperbaric bupivacaine in spinal anesthesia for caesarean section: a prospective, doubleblinded, randomized study. BMC anesthesiology. 18(1): 74.

4. Krishna TM, Panda N, Batra Y, Rajeev S. (2008) Combination of low doses of intrathecal ketamine and midazolam with bupivacaine improves postoperative analgesia in orthopedic surgery. European journal of anesthesiology. 25(4): 299-306.

5. Kirkham KR, Jacot-Guillarmod A, Albrecht E. (2018) Optimal Dose of Perineural Dexamethasone to Prolong Analgesia After Brachial Plexus Blockade: A Systematic Review and Meta-analysis. Anesthesthesia \& Analgesia. 126(1): 270-279.

6. Marhofer P, Columb M, Hopkins PM. (2018) Perineural dexamethasone: the dilemma of systematic reviews and meta-analyses. British journal of anesthesia. 120(2): 201-203.

7. Moeen SM, Moeen AM. (2017) Intrathecal dexamethasone vs. meperidine for prevention of shivering during transurethral prostatectomy: a randomized controlled trial. Acta Anesthesiologic Scandinavica. 61(7): 749-757.

8. Kroin JS, Schaefer RB, Penn RD. (2000) Chronic intrathecal administration of dexamethasone sodium phosphate: pharmacokinetics and neurotoxicity in an animal model. Neurosurgery. 46(1):178-82.

9. Sugita K, Kobayashi S, Yokoo A, Inoue T. (1983) Intrathecal steroid therapy for post-traumatic visual disturbance. Neurochirurgia (Stuttg). 26: 112-117.
10. Yanshuai M \& Shuang Q. (2017) Effects of dexmedetomidine in reducing postcesarean adverse reactions. Experimental and therapeutic medicine. 14(3): 2036-2039.

11. Wu HH, Wang HT, Jin JJ, Cui GB, Zhou KC, et al. (2014) Does dexmedetomidine as a neuraxial adjuvant facilitate better anesthesia and analgesia? A systematic review and meta-analysis. PLoS One. 9: e93114.

12. Safari F, Aminnejad R, Mohajerani SA, Farivar F, Mottaghi, et al. (2016) Intrathecal Dexmedetomidine and Fentanyl as Adjuvant to Bupivacaine on Duration of Spinal Block in Addicted Patients. Anesthesiology and pain medicine. 6(1): e26714.

13. Paramasivan A, Lopez-Olivo MA, Foong TW, Tan YW \& Yap AP. (2020) Intrathecal dexmedetomidine and postoperative pain: A systematic review and metaanalysis of randomized controlled trials. European Journal of Pain. 24(7): 1215-1227.

14. Albrecht E, Vorobeichik L, Jacot-Guillarmod A, Fournier N \& Abdallah FW. (2019) Dexamethasones superior to dexmedetomidine as a perineural adjunct for supraclavicular brachial plexus block: systematic review and indirect meta-analysis. Anesthesia \& Analgesia. 128(3): 543-554.

15. Elzayyat NS, Nagy HA \& Girgis K. (2014) Comparing the effect of adding dexmedetomidine versus dexamethasone on prolonging the duration of intrathecal bupivacaine in lower abdominal operations. Ain-Shams Journal of Anesthesiology. 7(3):388.

16. Sharma A \& Kumar R. (2019) Assessment of efficacy of Dexamethasone as an adjuvant to Bupivacaine for spinal anesthesia. Journal of Advanced Medical and Dental Sciences Research. 7(8): 218-222.

17. Zhang Y, Shan Z, Kuang L, Xu Y, Xiu H, et al. (2019) The effect of different doses of intrathecal dexmedetomidine on spinal anesthesia: A meta-analysis. International Journal of Clinical and Experimental Medicine. 9(10): 18860-18886.

18. Isguzar O, Baris S, Bozkurt A, Can B, Bilge S, et al. (2012) Evaluation of antinociceptive and neurotoxic effects of intrathecal dexmedetomidine in rats. Balkan Medical Journal. 29: 354-357. 
19. Gupta M, Gupta P \& Singh DK. (2016) Effect of 3 different doses of intrathecal dexmedetomidine (2.5microg, 5microg, and 10 microg) on subarachnoid block characteristics: a prospective randomized doubleblind dose-response trial. Pain Physician. 19: E411-420.

20. Ismaiel M, El-Safty O, El-Agamy A, Mohamed O \& Ali M. (2020) A comparative study between dexmedetomidine and dexamethasone as an intrathecal adjuvant for prevention of perioperative shivering in cesarean section. Ain-Shams Journal of Anesthesiology. 12(1): 1-9.

21. Hassan A, Abd-Allah Al-Kumite A, El-Deen A \& Shabaiek A. (2021) Clinical Comparative Study Between Intrathecal Dexmedetomidine and Dexamethasone on Prolonging the Duration of Intrathecal Blockade in Lower Limb Orthopedic Surgery. Al-Azhar Medical Journal. 50(2): 1467-1478.

22. Gao Z, Xiao Y, Wang Q \& Li Y. (2019) Comparison of dexmedetomidine and dexamethasone as adjuvant for ropivacaine in ultrasound-guided erector spinae plane block for video-assisted thoracoscopic lobectomy surgery: a randomized, double-blind, placebo-controlled trial. Annals of translational medicine. 7(22): 668.

23. Naziri F, Rabiee SM, Banihashem N, Hosseinjanzadeh K, Shirkhani Z, et al. (2013) Comparative study of intrathecal dexamethasone with epinephrine as adjuvants to lidocaine $\mathrm{n}$ cesarean section. Zahedan Journal of Research in Medical Sciences.15: 23-26.

24. Sakić L, Tonković D, Godan BJ \& Šakić K. (2015) The influence of dexamethasone administration in spinal anesthesia for femur fracture on postoperative cognitive dysfunction. Periodicum biologorum. 117(2): 281-285.

25. Sakić L, Tonković D \& Šakić K. (2019) Dexamethasone - Intrathecal Minimizer of Simple Haemathologic Stress Biomarkers in Hip Fracture. Acta clinica Croatica. 58(Supplement 1): 9-16.

26. Mazy A, Gad M \& Bedairy M. (2018) Preperitoneal postcesarean section bupivacaine analgesia: Comparison between dexamethasone and dexmedetomidine as adjuvants. Saudi Journal of Anaesthesia. 12(2): 183-189.

27. Gonapa DB \& Vanida DS. (2019) A comparative study between dexmedetomidine (1 $\mathrm{mcg} / \mathrm{kg})$ and dexamethasone $(0.1 \mathrm{mg} / \mathrm{kg})$ as adjuvants to $0.25 \%$ bupivacaine in caudal analgesia in pediatric patients undergoing lower abdominal surgeries-a prospective randomized comparative study. OSR Journal of Dental and Medical Sciences. 18(5): 45-52.

28. Song ZG, Pang SY, Wang GY \& Zhang Z. (2021) Comparison of postoperative analgesic effects in response to either dexamethasone or dexmedetomidine as local anesthetic adjuvants: a systematic review and meta-analysis of randomized controlled trials. Journal of anesthesia. 35(2):270-287.

29. Thakur J, Gupta B, Gupta A, Verma RK, Verma A, et al. (2019) A prospective randomized study to compare dexmedetomidine and dexamethasone as an adjunct to bupivacaine $\mathrm{n}$ transversus abdominis plane block for post-operative analgesia in caesarean delivery. International Journal of Reproduction, Contraception, Obstetrics and Gynecology. 8(12): 4904.

30. Sana S, Pathak RG \& Memon N. (2018) Duration of post-operative analgesia with Fascia Iliaca compartment block (ficb) using Bupivacaine with Dexmedetomidine and Bupivacaine with Dexamethasone in patients with proximal fracture femur. International Journal of Medical Science and Clinical Invention. 5: 3477-3486.

31. Gupta M, Gupta P \& Singh D. (2016) Effect of 3 different doses of intrathecal dexmedetomidine $(2.5 \mu \mathrm{g}$, $5 \mu \mathrm{g}$, and $10 \mu \mathrm{g}$ ) on subarachnoid block characteristics: A prospective randomized double-blind dose-response trial. Pain physician. 19: E411-E420.

32. Abdallah FW \& Brull R. (2013) Facilitatory effects of perineural dexmedetomidine on neuraxial and peripheral nerve block: a systematic review and metaanalysis. British journal of anesthesia. 110(6): 915-925.

33. Bani-Hashem N, Hassan-Nasab B, Pour EA, Maleh PA, Nabavi A, et al. (2011) Addition of intrathecal dexamethasone to bupivacaine for spinal anesthesia in orthopedic surgery. Saudi Journal of Anesthesia. 5: 382386.

34. Fayyaz MA, Khan AA \& Ali RL. (2015) Comparison between effect of bupivacaine and bupivacaine with dexamethasone on duration of analgesia in spinal anesthesia for elective caesarean section. Pakistan 
Journal of Medical \& Health Sciences. 9:979-982.

35. Dutta S, Gupta LK \& Sharma V. (2017) Evaluation of Efficacy of Dexamethasone as an Adjuvant to Bupivacaine for Spinal Anesthesia in Abdominal Surgery: An institutional Study. international Journal of Medical Research Professionals. 3(2): 419-422.

36. Shen QH, Li HF, Zhou XY, Yuan XZ \& Lu YP. (2020) Dexmedetomidine as an adjuvant for single spinal anesthesia in patients undergoing cesarean section: a system review and meta-analysis. Journal of international Medical Research. 48(5): 0300060520913423.
37. Thomas S \& Beevi S. (2006) Epidural dexamethasone reduces postoperative pain and analgesic requirements. Canadian Journal of Anesthesia. 53: 899-905.

38. Khafagy HF, Refaat A, El-Sabae HH \& Youssif MA. (2010) Efficacy of epidural dexamethasone versus fentanyl on postoperative analgesia. Journal of anesthesia. 24(4): 531-536.

39. Hong JY, Han SW, Kim WO, Kim EJ \& Kil HK. (2010) Effect of dexamethasone in combination with caudal analgesia on postoperative pain control in day-case paediatric orchiopexy. British journal of anaesthesia. 105(4): 506-510. 